\title{
INTEGRAL discovers a population of persistent intrinsically absorbed super-giant High-Mass X-Ray Binaries
}

\author{
R. Walter ${ }^{1} \dagger$, J. Zurita ${ }^{1}$, L. Bassani ${ }^{2}$, A. Bazzano ${ }^{3}$, A. Bodaghee ${ }^{1}$,

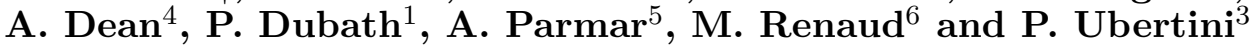 \\ ${ }^{1}$ INTEGRAL Science Data Centre, Chemin d'Ecogia 16, 1290 Versoix, Switzerland \\ ${ }^{2}$ IASF/CNR, Via Piero Gobetti 101, 40129 Bologna, Italy \\ ${ }^{3}$ IASF-INAF, Via Fosso del Cavaliere 100, 00133 Roma, Italy \\ ${ }^{4}$ School of Physics and Astronomy, University of Southampton, Highfield, S0171BJ, UK \\ ${ }^{5}$ ESTEC, Postbus 299, NL-2200 AG Noordwijk, The Netherlands \\ ${ }^{6}$ Service d'astrophysique, DAPNIA/DSM/CEA, 91191 Gif sur Yvette, France
}

\begin{abstract}
During the first year in operation, INTEGRAL has detected more than 28 new bright sources which emit the bulk of their emission above $10 \mathrm{keV}$. Follow-up observations of a subset of these sources in the X-ray band with XMM-Newton indicate that $80 \%$ of them are very strongly absorbed. More than half of these absorbed sources show strong pulsations with long periods ranging from 139 to 1300 s, i.e., they are slow X-ray pulsars. Many of these new sources are super-giant high-mass X-ray binaries (HMXB) in which the stellar wind of the companion star is accreted onto the compact object. The large local absorption in these new sources can be understood if the compact objects are buried deep in their stellar winds. These new objects represent half of the population of active super-giant HMXB.
\end{abstract}

Keywords. X-rays: binaries, gamma rays: observations, Galaxy: stellar content,stars: mass loss,stars: neutron,stars: binaries: close.

\section{A population of absorbed super-giant HMXB}

The INTEGRAL survey of the galactic plane and central regions has revealed the presence of few hundred sources in the energy range 20-100 keV. Twenty-five percent of the objects in the first INTEGRAL soft $\gamma$-ray Galactic plane survey catalog had no known counterparts and so their nature was unclear. The majority of those sources were detected in the Galactic bulge and Norma arm tangent region.

XMM-Newton follow-up observations of the first source discovered by INTEGRAL, IGR J16318-4848, revealed a spectrum with intense fluorescence lines (up to keV equivalent width) of $\mathrm{Fe} \mathrm{K} \alpha$ and $\mathrm{K} \beta$ and $\mathrm{Ni} \mathrm{K} \alpha$ as well as strong $\left(210^{24} \mathrm{~cm}^{-2}\right)$ low-energy absorption which makes the source extremely difficult to observe at energies below $4 \mathrm{keV}$. The strong absorption and unusual spectral properties led to the suggestion that IGR 16318-4848 is the first representative of a group of highly absorbed galactic binaries.

Here, we present the results of INTEGRAL, XMM-Newton and infrared observations of 10 among the new sources detected by INTEGRAL in the Galactic plane during the first year of the mission. Among those 10 sources we found.

- 7 intrinsically absorbed and persistent X-ray emitters. Five of them are pulsars featuring long (139-1300 sec) spin periods. Orbital periods (<10 days) are known for two objects in which eclipses could be detected. Five sources have early type stellar companions i.e. they are HMXB. The long spin periods and short orbital periods indicate

$\dagger$ email: Roland.Walter@obs.unige.ch 
that the sources are very likely super-giant systems. Since the X-ray properties of all sources are very similar all those sources are likely persistent super-giant HMXB systems.

- 2 intrinsically absorbed transient super-giant HMXB.

- 1 unabsorbed persistent LMXB.

Detailed studies of the other new INTEGRAL sources are needed to confirm that a significant fraction of them are persistent absorbed super-giant systems. In addition, INTEGRAL has also discovered several new transient super-giant systems that should be slightly different objects. In any case, INTEGRAL has already almost doubled the number of active super-giant HMXB known in the Galaxy.

\section{Source geometry}

The observed spectra provide several clues about the source geometry:

- The continuum spectral shape is best characterized by a transmission geometry rather than by reflection. Fe line with keV apparent equivalent width are also not compatible with reflection. This indicates that the compact sources are embedded in dense material.

- The absence of reddening in excess to the galactic value in the infrared counterparts indicates that the absorption is local to the sources.

- The centroids of the Fe lines indicate low Iron ionization i.e. that the region where fluorescence takes place is larger than $10^{7} \mathrm{~km}$. The absence of modulation of the Fe line on the spin period indicate that the fluorescence region is larger than $10^{8} \mathrm{~km}$. The fluorescence/absorbing region is not associated to the accretion column nor with an accretion disk, its size is comparable to or larger than the orbital radius.

- The relation between the fluorescence line flux and the absorbing column density is as expected for a spherical geometry. The fluorescence region is not extremely patchy.

- Variable absorbing column densities or Iron line fluxes observed in few objects indicate that most of the absorbing/fluorescence region lies within $10^{8-9} \mathrm{~km}$ (IGR J163184848, IGR J16320-4751) or within a few orbital radii (IGR J17252-3616).

The source persistency and the strong absorption could be understood if the compact objects orbit continuously in a dense component of the stellar wind. Short orbital periods are expected and observed. High column densities could be related with slow dense stellar winds in the orbital plane. The lack of wind acceleration could be related to the photoionization of the X-ray sources.

\section{Conclusion}

INTEGRAL has unveiled a population of super-giant HMXB of long spin periods and short orbital periods in which the compact objects are orbiting within a dense component of the super-giant stellar wind. Such systems are characterized by persistent (but variable) hard X-ray emission.

\section{References}

Bodaghee, et al., 2005, A\&A submitted

Hill, et al., A\&A, A\&A in press

Rodriguez, et al., 2005, MNRAS submitted

Walter, et al., 2003, A\&A 411, 427
Walter, et al., 2004, ESA-SP 552, 417

Walter, et al., 2005, A\&A submitted

Zurita, et al., 2005, A\&A submitted 